\title{
EL REGISTRO ARQUEOBOTÁNICO EN EL VALLE DEL SALADO, MENDOZA: PROBLEMAS TAFONÓMICOS Y EXPLOTACIÓN HUMANA
}

\author{
THE ARCHAEOBOTANICAL RECORD FROM EL SALADO VALLEY, MENDOZA: \\ TAPHONOMIC PROBLEMS AND HUMAN EXPLOITATION
}

\author{
Carina Llano ${ }^{1}$ y Gustavo Neme ${ }^{1}$
}

\begin{abstract}
El objetivo del presente estudio es dar a conocer los resultados de la identificación taxonómica de los macrorrestos vegetales recuperados en las excavaciones arqueológicas llevadas a cabo en el valle del Salado, Malargüe, Mendoza (Argentina), discutiendo aspectos vinculados al origen de las muestras y al uso de las especies identificadas por parte de las poblaciones humanas. Los macrorrestos estudiados fueron recuperados durante las excavaciones de los sitios arqueológicos Puesto Jaque II (PJ-II) y Gendarmería Nacional 5 (GN-5). Temporalmente los sitios corresponderían a los últimos 1000 años a.p. La identificación taxonómica de los restos arqueobotánicos se basó en el análisis morfológico comparativo de macrorrestos (frutos y semillas) actuales y arqueológicos. Los resultados sugieren un origen diferencial de las muestras de ambos sitios arqueológicos. Así, mientras que por un lado la muestra obtenida en PJ-II tendría un origen principalmente antrópico, con el consumo de especies tales como Schinus polygamus (Cav.) Cabrera, Berberis empetrifolia Lam. y probablemente Oxalis sp. y Chenopodium sp., por el otro, la muestra proveniente de GN-5 habría tenido un origen natural. En el trabajo se concluye que es poco probable que las dos últimas especies mencionadas hayan sido consumidas (aunque no se descarta esta posibilidad), siendo su estatus diferente al de las tres especies previamente mencionadas.
\end{abstract}

Palabras claves: arqueobotánica, ambientes de altura, subsistencia, tafonomía.

This paper presents the results of taxonomic identification of the macroremains recovered from archaeological sites in the El Salado valley, Malargüe, Mendoza (Argentina), discussing aspects related to the origin of the samples and the use of the identified species by human populations. The macroremains were recovered from the sites of Gendarmería Nacional-5 (GN-5) and Puesto Jaque-II (PJ-II). The sites correspond to the last 1,000 years BP. The taxonomic identification of archaeobotanical remains was based on comparative morphological analysis (fruits and seeds) with archaeological and contemporary macroremains. The results suggest a different origin for the samples from these two sites. While on one hand the sample from PJ-II had an anthropogenic origin, suggesting the consumption of species such as Schinus polygamus (Cav.) Cabrera, Berberis empetrifolia Lam., and probably, Oxalis sp., and Chenopodium sp. On the other hand, it appears that the GN-5 sample was deposited by natural causes. We conclude that it is unlikely that the last two mentioned species were consumed (but do not rule this possibility), as their status was different from the three earlier mentioned species.

Key words: Archaeobotany, high altitude environments, subsistence, taphonomy.

Los trabajos arqueológicos en las regiones de altura del sur de Mendoza han apuntado a abordar diferentes tipos de problemáticas tales como la historia cultural de la región, el uso diferencial del paisaje, la movilidad, el intercambio y las estrategias de subsistencia de los grupos humanos con sus cambios a través del tiempo (Durán 2004; Gil et al. 2006; Lagiglia 1997; Lagiglia et al. 1994; Neme 2007; Neme y Gil 2008). Estos trabajos han incluido la discusión de distintos tipos de materiales entre los que se cuentan los estudios de arqueofauna (Gil y Neme 2002), de material lítico (Pérez Winter
2008), bioantropológicos (Novellino y Neme 1999) e isotópicos de $\delta^{13} \mathrm{C}$ y $\delta^{15} \mathrm{~N}$ sobre material óseo, tanto de origen animal como humano (ver más detalles en Gil et al. 2006), entre otros. Pese a que muchas de las discusiones implicaron cuestiones vinculadas con la subsistencia, haciéndose referencia directa o indirecta al registro vegetal, solo recientemente se ha incluido en los estudios arqueológicos regionales esta línea de evidencia (Llano 2008).

La arqueobotánica ha sido definida como una disciplina que se centra en el análisis de las interacciones entre el hombre y las plantas, a través

1 CONICET-Departamento de Antropología, Museo de Historia Natural de San Rafael, Parque Mariano Moreno (5600) San Rafael, Mendoza, Argentina. carina_lourdes@yahoo.com.ar; gustavoneme@arqueologiamendoza.org 
del registro arqueológico (Hastorf 1999). En este sentido, en el presente trabajo se considera como apropiado el uso del término arqueobotánica, dado que las prácticas a llevarse a cabo están estrechamente vinculadas con objetivos y preguntas de corte netamente arqueológico, las cuales requieren la identificación e interpretación del registro vegetal (Giovannetti et al. 2008). La creciente importancia que ha tenido la disciplina en las últimas décadas ha permitido mejorar la reconstrucción de los sistemas de subsistencia humanos del pasado. Al igual que otros aspectos del registro arqueológico, el uso de los macrorrestos vegetales para reconstruir el pasado tiene sus desventajas, debido a que el material que se preserva es una muestra del conjunto total, $\mathrm{y}$ es afectado por numerosos factores conocidos y desconocidos de depositación, preservación y recuperación (Johannessen 1988). De esta forma, la presencia y ausencia de macrorrestos pueden ser consecuencia conjunta de la acción de elementos humanos y no humanos (Hastorf y Popper 1988). A pesar de estas desventajas, el estudio de los macrorrestos vegetales tiene varias cualidades que convierten su análisis en una herramienta útil para investigar tanto aspectos económicos como sociales de las poblaciones humanas (Johannessen 1988; Pochettino y Scattolin 1991; Rodríguez 1999), su interacción con el medio y los cambios en la estructura ambiental de un determinado lugar (Jones 1991; Minnis 1978; Rodríguez 2005).

El presente trabajo tiene como objetivos dar a conocer los resultados de la identificación taxonómica de los macrorrestos vegetales recuperados en sitios arqueológicos del valle del Salado (Malargüe, Mendoza, Argentina), explorar la incidencia de factores no humanos en la conformación del registro arqueobotánico y a partir de éstos abordar la discusión acerca del uso dado a las plantas por parte de las poblaciones humanas de dicho valle en los últimos mil años. El análisis de los macrorrestos aquí estudiados proviene de las excavaciones realizadas en dos sitios arqueológicos: Gendarmería Nacional 5 (GN-5) y Puesto Jaque II (PJ-II).

\section{Descripción del Área de Estudio}

\section{Fitogeografía}

La ubicación geográfica de Mendoza y sus contrastes topográficos entre la Cordillera de los Andes hacia el oeste y las extensas llanuras hacia el este, determinan una gran variedad de ambientes, lo cual se refleja en la diversidad florística (Roig et al. 2000).

A una escala regional, el clima del valle del Salado (afluente del río Atuel) se encuentra influenciado por las precipitaciones del anticiclón del Pacífico, el cual es responsable de las nevadas invernales en la alta cordillera (Capitanelli 2005). El anticiclón del Atlántico llega de manera atenuada, por lo cual solo tiene escaso efecto en la parte baja del sector cordillerano del Atuel, a través de sus precipitaciones veraniegas (Capitanelli 2005).

Fitogeográficamente, el área corresponde al Dominio Andino, representado por la Provincia Altoandina y Patagónica (Cabrera 1976). Roig (1972) incluye a este tipo de vegetación dentro de las formaciones de montaña, la cual es discriminada de acuerdo con los niveles altitudinales. A partir de los $1.500 \mathrm{msm}$ hasta los $2.300 \mathrm{msm}$ hay una mayor proporción de especies pertenecientes a la provincia Patagónica, que hasta los $1.800 \mathrm{msm}$ se mezclan con elementos de la provincia del Monte, y desde los $2.300 \mathrm{msm}$ hasta los $3.800 \mathrm{msm}$ predominan los elementos de la provincia Altoandina (Böcher et al. 1972). La vegetación de la provincia Altoandina está representada por Adesmia abobata Clos, yaretas o plantas en cojín como Mulinum crasifolium Phil. y Adesmia subterranea Clos, otras especies de este tipo son Oxalis bryoides Phil., Verbena minutiflora Briq. ex Moldenke, Azorella mucamentacea (Phil.) Hauman. En la provincia Patagónica la vegetación está caracterizada por la presencia de Adesmia pinifolia Gillies ex Hook \& Arn, hay coironales de Stipa tenuisima Trin., Stipa speciosa Trin. \& Rupr. y Stipa chrysofila E. Desv. (Böcher et al. 1972; Roig 1972). En una escala más acotada, la vegetación del área de estudio comparte condiciones y características tanto de la franja ecológica de la "vegetación de montaña" como de la "vegetación de la estepa patagónica". La vegetación de montaña es dominada por comunidades arbustivas en la base y parte media, las cuales son reemplazadas paulatinamente por pastizales en las zonas más altas. La vegetación que representa a la estepa patagónica muestra un dominio de especies tales como Stipa spp., Mulinum spinosum (Cav.) Pers, Adesmia pinifolia Gillies ex Hook \& Arn, Ephedra ochreata Miers, Berberis empetrifolia Lam., Schinus polygamus (Cav.) Cabrera; entre otras. 


\section{Los sitios Gendarmería Nacional y Puesto Jaque}

Las excavaciones arqueológicas se concentraron en las áreas de los valles intermedios de cordillera a unos $2.000 \mathrm{msm}$ (Neme 2007), en donde se realizaron transectas superficiales paralelas al cauce del río Salado. A lo largo de éstas, se detectaron una serie de concentraciones de materiales arqueológicos (principalmente líticos) dispuestos en forma continua, de las cuales se seleccionaron dos para ser excavadas: Gendarmería Nacional $5(\mathrm{GN}-5)$ y Puesto Jaque II (PJ-II).

GN-5 es un sitio a cielo abierto cuyas coordenadas son $35^{\circ} 11^{\prime} 32^{\prime \prime} \mathrm{S}, 70^{\circ} 01^{\prime} 39^{\prime \prime} \mathrm{O}$ y su altura de $2.006 \mathrm{msm}$ (Figura 1), el cual se excavó por su condición topográfica y la mayor densidad de materiales superficiales. Pese a que los materiales extraídos aún no han sido analizados en su totalidad, se advierte la ausencia de cerámica, de molinos y manos, como así también de instrumentos formales (solo aparecen lascas con retoques). La mayor parte del conjunto está constituida por desechos líticos y escaso material óseo muy meteorizado. La baja densidad de materiales (143,2 hallazgos por $\mathrm{m}^{3}$ ) en relación a otros sitios de la región, así como el tipo de evidencias recuperadas, sugiere que GN-5 habría sido ocupado por grupos de cazadores-recolectores durante lapsos temporales cortos. El análisis preliminar sugiere que las tareas desarrolladas en el mismo se habrían limitado al reacondicionamiento de instrumentos y al procesamiento de alimentos de origen animal.

El segundo sitio analizado, PJ-II, se encuentra ubicado sobre la margen derecha del río Salado. Este sitio arqueológico está emplazado sobre una geoforma en forma de $\mathrm{U}$, rodeada de una barda de conglomerados que conforman una pequeña cuenca de sedimentos, localizado a $35^{\circ} 10^{\prime} 42^{\prime \prime} \mathrm{S}$, $69^{\circ} 59^{\prime} 07^{\prime \prime} \mathrm{O}$ a una altura de $1.970 \mathrm{msm}$ (Figura 1). Los hallazgos incluyen cerámica, raspadores, puntas de proyectil, desechos de talla, material óseo en muy mal estado de conservación y una importante cantidad de macrorrestos vegetales. Durante las excavaciones en PJ-II se recuperaron un total de 11 elementos de molienda en estratigrafía, lo que representa una elevada densidad para los estándares de la región (Neme 2007). Trabajos previos ya destacan la alta frecuencia de equipamiento de molienda en el mismo valle a menos de 10 kilómetros de los sitios estudiados (Schobinger 1975). La alta densidad de materiales recuperados (301 hallazgos por $\mathrm{m}^{3}$ ), sumada a la diversidad de instrumentos, la presencia de cerámica y restos óseos, hace que PJ-II sea caracterizado como un sitio de cazadoresrecolectores que desarrollaron actividades múltiples durante lapsos temporales más prolongados que los de GN-5. De forma preliminar los estudios sugieren que las actividades habrían sido la confección de instrumentos, así como el procesamiento y consumo de vegetales y animales, y probablemente la confección de cerámica.

Para conocer la antigüedad de las ocupaciones humanas se enviaron muestras de carbón, las cuales fueron datadas por AMS en los últimos 1000 años a.p. GN-5 abarca un lapso desde los $871 \pm 38$ a $557 \pm 38$ años a.p., mientras que PJ-II fue fechado entre $646 \pm 42$ a $357 \pm 35$ años a.p. (Tabla 1). Como se detalla más adelante, ambos sitios arqueológicos tienen una potencia de sedimento que supera el metro de profundidad. La ocurrencia de fechados tardíos confirma lo observado en otros sectores del valle del Salado, por lo que la tasa de depositación, al menos en algunos tipos de geoformas, es muy alta.

Durante los trabajos de campo se detectó en ambas excavaciones la presencia de alteraciones postdepositacionales causadas por animales fosoriales (Figura 2). De la gran cantidad de galerías,

Tabla 1. Cronología de los sitios arqueológicos analizados. Chronology from the archaeological sites analyzed.

\begin{tabular}{lcccccc}
\hline Sitio & Cuadrícula & Nivel & Fechado años a.p. & Calibrado años AD & Material & Código \\
\hline GN-5 & S-1 & 6 & $871 \pm 38$ & $1152-1277$ & Carbón & AA66570 \\
GN-5 & S-1 & 22 & $557 \pm 38$ & $1327-1339 / 1390-1451$ & Carbón & AA66571 \\
PJ-II & A-1 & 4 & $357 \pm 35$ & $1479-1643$ & Carbón & AA66575 \\
PJ-II & A-2 & 12 & $646 \pm 42$ & $1296-1411$ & Carbón & AA66574 \\
PJ-II & A-2 & 20 & $395 \pm 37$ & $1457-1626$ & Carbón & AA66573 \\
\hline
\end{tabular}




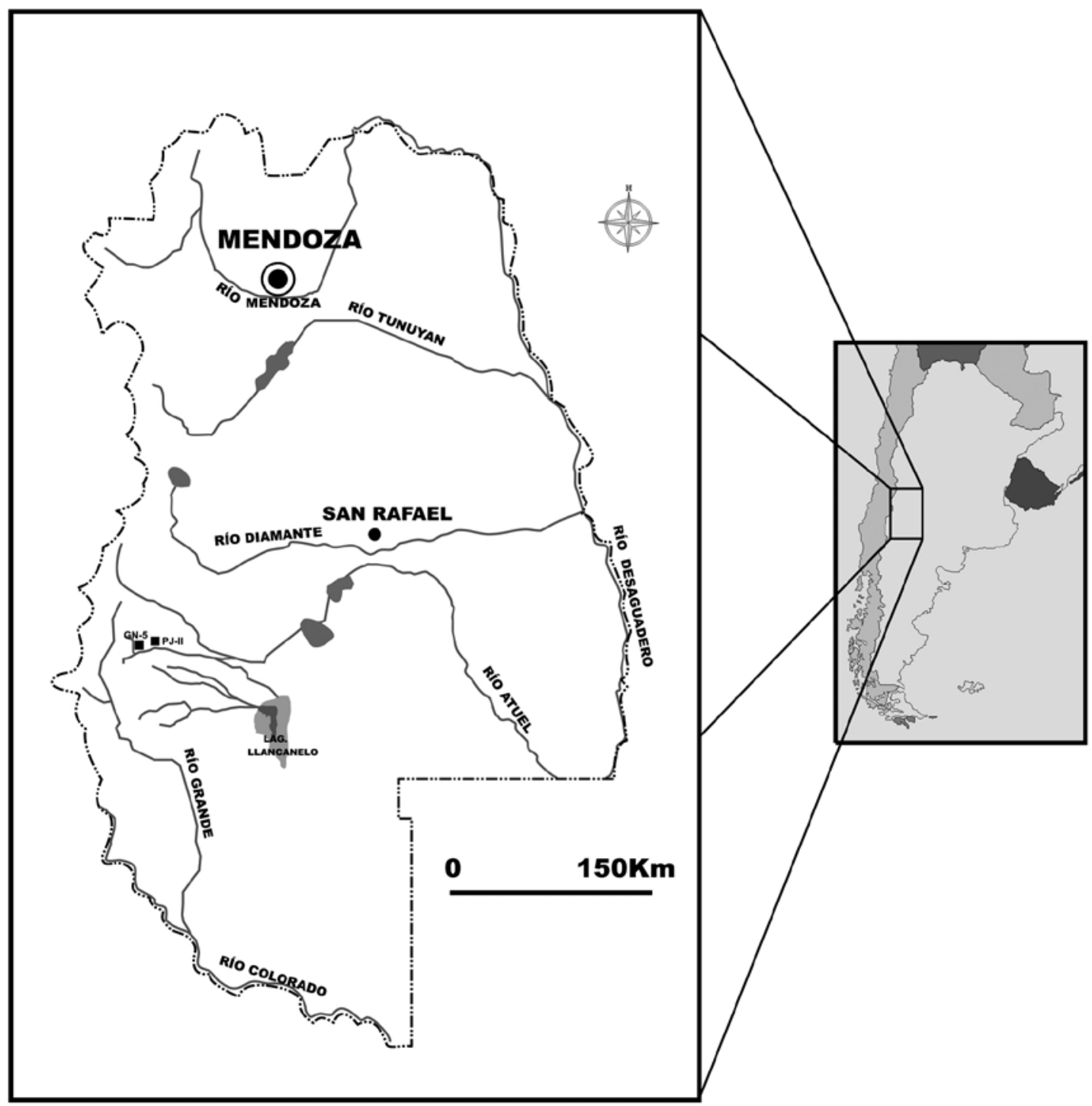

Figura 1. Localización de los sitios arqueológicos en el Valle del Salado.

Location of the archaeological sites in El Salado valley.

cuyo sedimento fue extraído y cernido por separado, se recuperaron partes esqueletarias de estos microvertebrados y en particular en el sitio PJ-II se obtuvieron macrorrestos vegetales.

La presencia de estas galerías de roedores sugiere que éstos habrían sido los principales causantes de la remoción de sedimentos y materiales culturales en ambos sitios arqueológicos. De esta forma es altamente probable que la inversión de fechados obtenidos (Tabla 1) pueda ser explicada por este tipo de bioturbación. Sin embargo, dado que los fechados no muestran un amplio rango temporal de ocupación en ambos sitios (ca. 300 años en cada uno), en este trabajo se considerará a cada uno de ellos como sitios unicomponentes.

\section{Materiales y Métodos}

Los métodos de recuperación del material arqueobotánico utilizados fueron la flotación asistida con máquina, cuyo tamaño de malla para recuperar la fracción pesada fue de 1 milímetro (Badal et al. 2003; Alonso Martínez et al. 2003; Pearsall 1989), y la extracción en zaranda durante la excavación. 


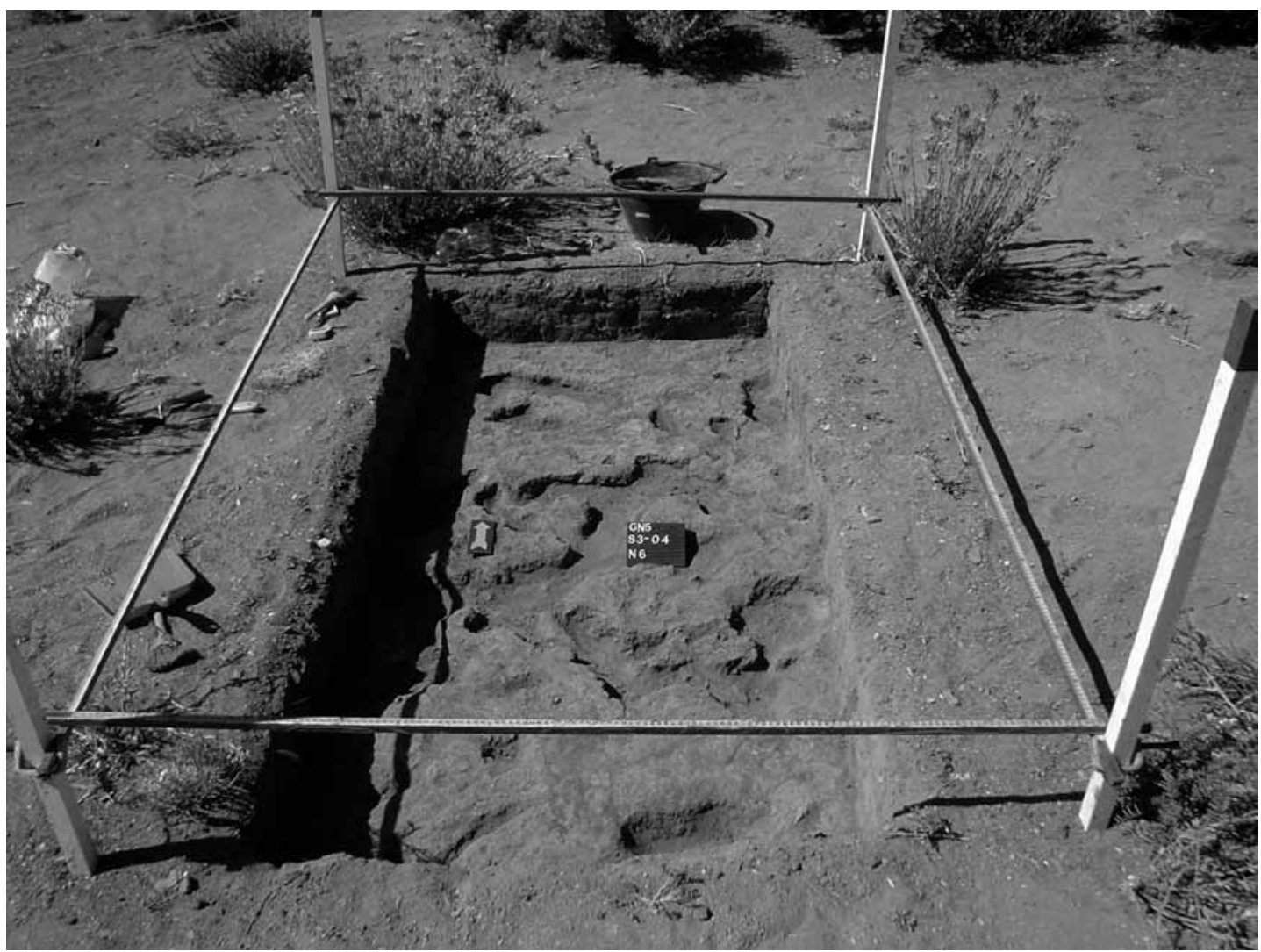

Figura 2. Excavación de GN-5 reflejando las bioturbaciones generadas por microvertebrados. Bioturbations in GN-5 generated by microvertebrates.

En cuanto al tamaño de la malla utilizada para la recuperación con zaranda fue de 1 milímetro, cerniéndose todo el sedimento extraído. El material recuperado se extrajo con pinza y fue guardado en bolsas herméticas, conservando las referencias de la excavación. En el sitio GN-5 se excavaron dos cuadrículas de dos metros por uno (S-1 y S-2) alcanzando profundidades de $145 \mathrm{~cm}$ en la primera y $125 \mathrm{~cm}$ en la segunda. Los materiales aquí presentados corresponden a la cuadrícula S-2. En el sitio PJ-II se excavaron tres cuadrículas contiguas en forma de trinchera (A-1, A-2 y A-3) de dos metros por dos metros cada una, con una dirección Este-Oeste. En las cuadrículas A-1 y A-2 se excavó hasta alcanzar el nivel de rocas a los $105 \mathrm{~cm}$ (nivel 21) y en A-3 se abandonó la excavación en el nivel 13. En este caso, los materiales analizados corresponden a las cuadrículas A-1 y A-2.

Para ambas excavaciones se siguieron niveles artificiales de $5 \mathrm{~cm}$, en cada uno de los cuales se tomó una muestra de 5 litros de sedimento para flotación. El muestreo realizado fue sistemático recolectando el sedimento de forma dispersa por toda la unidad estratigráfica o nivel, tanto en su extensión como en su potencia, de manera que la muestra extraída fuera representativa del conjunto. De esta manera, se obtiene una visión integrada del sitio bajo estudio, que mediante la cuantificación de los resultados permite la comparación inter e intrasitio (Pearsall 1989). El volumen total de material flotado del sitio GN-5 fue de 55 litros de sedimento provenientes de la cuadrícula S-2. Para el sitio PJ-II se flotaron un total de 55 litros de sedimento provenientes de la cuadrícula A-1; y 95 litros de sedimento provenientes de la cuadrícula A-2. Si bien las muestras de flotación se tomaron en todos los niveles estratigráficos, parte de las mismas se rompieron durante el traslado, por lo cual no fueron consideradas en el análisis.

Para el presente trabajo, el registro arqueobotánico analizado está conformado por semillas y endocarpos enteros y fragmentados. Los carporrestos se encontraron en diferentes estados de preservación (seco y/o carbonizado), incluyéndose en la 
muestra bajo estudio la totalidad de los mismos. En el acápite de resultados se resumen el estado de preservación y estructural de los carporrestos recuperados. Cabe mencionar que en este estudio aún no han sido analizados macrorrestos (carbón) como así tampoco microrrestos (almidón y fitolitos), los cuales serán procesados en trabajos posteriores.

El análisis de laboratorio comenzó con la separación del material de la fracción liviana de la flotación (FL), a través del cernido en tamices de $2 \mathrm{~mm}$ y $0,4 \mathrm{~mm}$. La separación en dos partes de la muestra de flotación ayuda al momento de la observación con microscopio estereoscópico, evitando tener que reenfocar por cada fracción de material analizada. Después de que la muestra fue dividida, cada fracción fue examinada individualmente, separando a ojo desnudo el material $>2 \mathrm{~mm}$, mientras que la fracción $<2 \mathrm{~mm}$ se examinó íntegramente bajo microscopio estereoscópico Nikon SMZ800.

La identificación de las especies vegetales se llevó a cabo por comparación anatómica del material arqueológico con respecto a las especies actuales que forman parte de la colección de referencia. La misma está integrada por las especies vegetales coleccionadas en el área de estudio, en los alrededores de los sitios arqueológicos. Además, se dispuso de material del Herbario del Instituto de Botánica Darwinion (IBODA), del Herbario de Ruiz Leal (CCyT) y del Departamento de Botánica del Museo de Historia Natural de San Rafael (MHNSR). Los caracteres cualitativos evaluados en la morfología externa de los macrorrestos fueron: color, forma, tamaño, ornamentación de la cubierta, posición del hilo, entre otros (Martin y Barkley 1973). Cuantitativamente se tuvieron en cuenta los siguientes caracteres biométricos: largo, ancho y grosor (Cowan 1997; Gremillion 1993, 1997). Otro parámetro que se tuvo en cuenta es el índice de forma, que se expresa en largo sobre ancho. Este índice refleja el grado de longitud o de ancho del órgano bajo estudio (semilla, fruto, etc.).

Se emplearon cantidades absolutas, ubicuidad y densidad de taxa para la cuantificación de los macrorrestos (Jones 1991; Miller 1988; Popper 1988). El propósito de la descripción numérica es una base para inferir el comportamiento humano pasado, para hacer reconstrucciones ambientales o para explicar los procesos de formación de sitio (Jones 1991).

\section{El origen de la muestra estudiada}

Teniendo en cuenta que no sólo la conducta humana actúa en la formación del registro arqueobotánico y que distintos factores tales como la conservación diferencial y la cantidad de semillas que produce una planta son, entre otras, las variables a tener en cuenta al momento de cuantificar e interpretar el registro arqueobotánico (Buxó 1997; Keepax 1977; Minnis 1981), se utilizaron métodos de análisis que ayudaron a diferenciar los posibles orígenes de los carporrestos encontrados en los sitios. De esta forma, los trabajos de campo incluyeron además, la realización de dos sondeos en las proximidades de cada uno de los sitios analizados. El objetivo de los mismos fue controlar la forma en que las plantas locales se dispersan naturalmente sin intervención humana, aislando de este modo variables que ayuden a identificar el impacto de la actividad antrópica en el sitio (Keepax 1977; Miksicek 1987; Minnis 1981). Para los sondeos, la metodología utilizada fue la excavación de un pozo de $40 \times 40 \mathrm{~cm}$ de donde se extrajeron niveles artificiales de $10 \mathrm{~cm}$ llegando hasta el nivel de rodados que impedían seguir con los mismos. Los sedimentos recuperados fueron flotados en laboratorio, procesando un total de 20 litros en cada uno de los sondeos. La decisión de tomar niveles arbitrarios de $10 \mathrm{~cm}$ para el sondeo se debe al tipo de información que se quiere relevar (diversidad florística, presencia/ausencia de taxa), en tanto que para la excavación la toma de niveles arbitrarios de $5 \mathrm{~cm}$ atiende a controlar factores tales como la formación del sitio y distribución espacial de los materiales, entre otros.

\section{Resultados del Análisis Arqueobotánico}

\section{Determinación taxonómica y análisis cuantitativo}

La muestra total de macrorrestos del sitio GN-5 se encontraba en estado seco y en general presentaba un buen estado de conservación. Solo se recuperó material de la fracción liviana de la flotación, en tanto que de la fracción pesada y de zaranda no se obtuvieron macrorrestos. De la fracción liviana se recuperaron un total de 122 macrorrestos (Tabla 2), determinados en diferentes niveles taxonómicos. En la Tabla 3 se resumen las variables tomadas en el análisis de los carporrestos. Se determinaron 


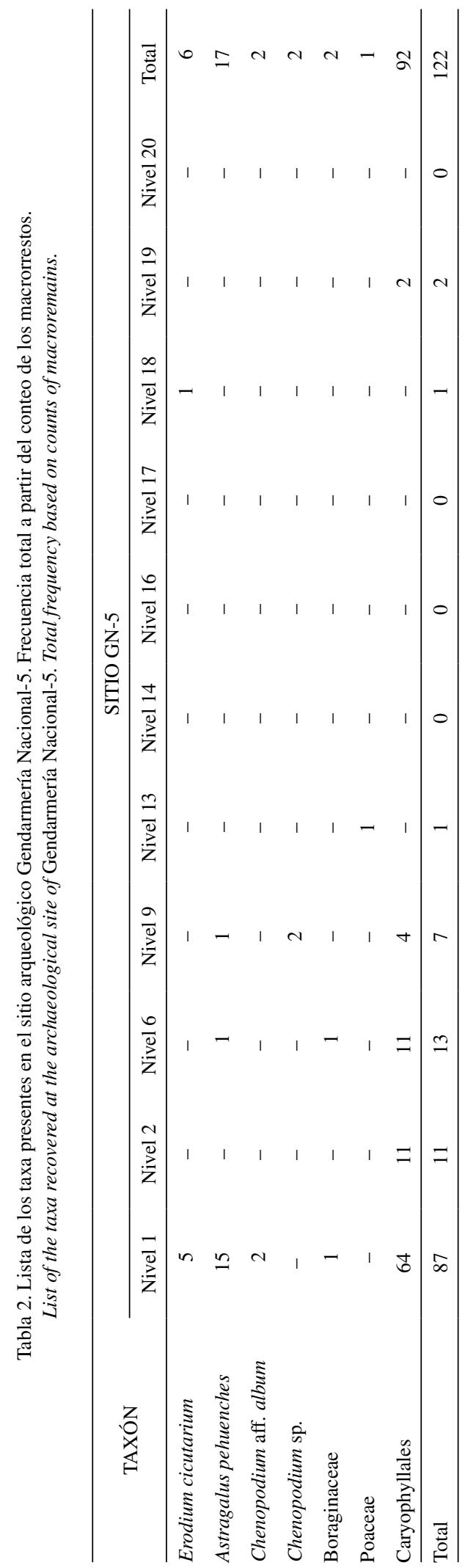

los siguientes taxa: Caryophyllales, Poaceae, Boraginaceae, Chenopodiaceae. Dentro de esta familia se encontró Chenopodium aff. album y Chenopodium sp. La familia Fabaceae está representada por Astragalus pehuenches Niederl. conocida vulgarmente como "yerba loca", y la familia Geraniaceae se encuentra representada por Erodium cicutarium (L.) H'Hér. ex Aiton, conocida vulgarmente como alfilerillo (Tabla 2).

Para describir la tendencia cuantitativa se calculó la densidad y ubicuidad de los restos recuperados (Buxó 1997; Hastorf y Popper 1988; Jones 1991). El análisis de densidad mostró que la mayor cantidad de especímenes por litro está dada por el orden Caryophyllales (Figura 3a), con una diferencia importante en relación a los taxa restantes; Astragalus pehuenches es el segundo taxa en número de ejemplares. De la misma forma, el resultado del análisis de ubicuidad muestra que el taxón más ubicuo es Caryophyllales, seguido del taxón Astragalus pehuenches. Los taxa restantes tienen valores similares, siendo mayores para el caso de Boraginaceae y Erodium cicutarium (Figura 3b).

En el análisis del sitio PJ-II se tuvieron en cuenta tanto los macrorrestos recuperados de zaranda como los de la fracción liviana de la flotación, sumando un total de $\mathrm{n}=1.537$ macrorrestos, de los cuales el $31 \%$ de la muestra total se encuentra carbonizada ( $\mathrm{n}=480$ macrorrestos). Se determinaron los siguientes taxa: Caryophyllales, Poaceae, Anacardiaceae, encontrándose la especie Schinus polygamus (Cav.) Cabrera, conocida con el nombre vulgar de "Molle", la familia Berberidaceae, encontrándose Berberis empetrifolia Lam. conocida con el nombre vulgar de "calafate, michay", la familia Chenopodiaceae (Chenopodium aff. papulosum), la familia Geraniaceae (Erodium cicutarium), y la familia Oxalidaceae, encontrándose Oxalis sp. (Tabla 4). En la Tabla 5 se presentan las variables tomadas en el análisis de los carporrestos. Para calcular la densidad se tomó solamente la muestra proveniente de la flotación, ya que la de zaranda no especifica el volumen de sedimento procesado. Ambas cuadrículas, A-1 y A-2, mostraron que el taxón más denso es Caryophyllales (Figura 4a), que si bien no se pudo avanzar más en su identificación, todo el material corresponde al mismo taxón. El resto de los taxa presentan valores de densidad similares en ambas cuadrículas, destacándose Schinus polygamus y Chenopodiaceae (Figura 4a). La ubicuidad refleja que el taxón presente en la 

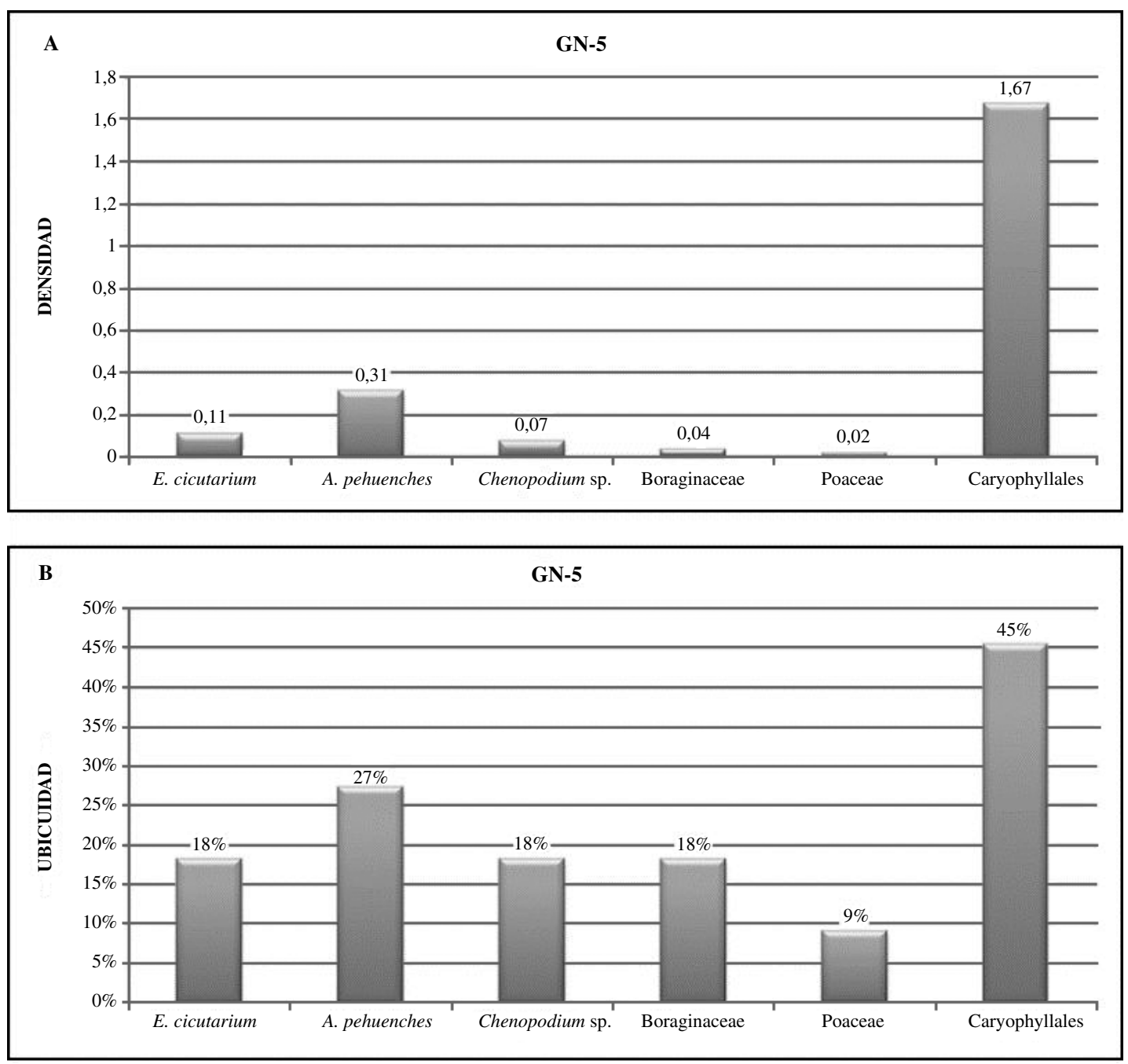

Figura 3. (A) Densidad de los macrorrestos vegetales en el sitio GN-5; (B) ubicuidad de los macrorrestos vegetales en el sitio GN-5. (A) Density of plant remains from $G N-5$ sites; (B) ubiquity of plant remains from $G N-5$ sites.

mayor parte de las muestras de ambas cuadrículas es Schinus polygamus seguido por Caryophyllales y Chenopodiaceae (Figura 4b).

Para el caso de los dos sondeos de prueba, los análisis de densidad mostraron que el taxón que presenta los valores más altos es Caryophyllales (Figura 5a), tanto para GN-5 como para PJ-II. Los valores de ubicuidad en el sondeo de GN-5 reflejan un alto porcentaje para Caryophyllales y Erodium cicutarium, mientras que en el caso del sondeo de PJ-II sólo se recuperaron restos correspondientes al orden Caryophyllales y a la especie Oxalis sp., presentando ambos taxa un alto porcentaje (Figura $5 b$ ).

\section{Discusión}

Los resultados del análisis de los macrorrestos recuperados de los sitios GN-5 y PJ-II pueden ser utilizados para evaluar las estrategias vinculadas a la explotación de recursos por parte de las poblaciones humanas que habitaron el valle del Salado, y proveer información sobre los procesos de formación del registro arqueológico. Las inferencias que se realizaron sobre los conjuntos analizados dependieron de la especificidad con la que se lograron determinar los macrorrestos, por lo que en algunos casos las interpretaciones sobre la interacción Hombre-planta se vieron limitadas. 
Tabla 3. Estado de conservación, estructural y densidad de los restos arqueológicos vegetales recuperados mediante flotación. Plant part, condition, structure and density of archaeological remains recovered by flotation in the archaeological site GN-5.

\begin{tabular}{cccccc}
\hline Nivel & Taxa & Parte Vegetal & Estado de Conservación & Estado Estructural & Densidad (No/5 litros) \\
\hline \multirow{2}{*}{1} & Erodium cicutarium & Fruto & Seco & Entero & 1 \\
& Astragalus pehuenches & Semilla & Seco & Entero & 3 \\
& Chenopodium sp. & Semilla & Seco & Entero & 0,4 \\
& Caryophyllales & Semilla & Seco & Entero & 12,8 \\
& Boraginaceae & Semilla & Seco & Entero & 0,2 \\
\hline \multirow{2}{*}{2} & Caryophyllales & Semilla & Seco & Entero & 2,2 \\
\hline \multirow{2}{*}{6} & Astragalus pehuenches & Semilla & Seco & Entero & 0,2 \\
& Boraginaceae & Semilla & Seco & Entero & 0,2 \\
\hline \multirow{2}{*}{9} & Caryophyllales & Semilla & Seco & Entero & 2,2 \\
\hline 13 & Astragalus pehuenches & Semilla & Seco & Entero & 0,2 \\
\hline 18 & Chenopodium sp. & Semilla & Seco & Entero & 0,4 \\
\hline 19 & Caryophyllales & Semilla & Seco & Entero & 0,8 \\
\hline
\end{tabular}

Tabla 4. Lista de los taxa presentes en el sitio arqueológico Puesto Jaque-II. Frecuencia total a partir del conteo de los macrorrestos. $(*)$ Macrorrestos carbonizados.

List of the taxa recovered from the archaeological site of Puesto Jaque-II. Total frequency based on counts of the macroremains. (*) Charred macroremains.

\begin{tabular}{|c|c|c|c|c|}
\hline \multirow{3}{*}{ TAXÓN } & \multicolumn{4}{|c|}{ SITIO PJ-II } \\
\hline & \multicolumn{2}{|c|}{ Zaranda } & \multicolumn{2}{|c|}{ Flotación } \\
\hline & A-1 & A-2 & A-1 & A-2 \\
\hline Schinus polygamus & $197^{*}$ & $198^{*}$ & $35^{*}$ & $41^{*}$ \\
\hline Berberis empetrifolia & - & $3^{*}$ & $3^{*}$ & - \\
\hline Erodium cicutarium & - & - & - & 4 \\
\hline Oxalis sp. & - & - & 1 & - \\
\hline Chenopodium aff. papulosum & - & - & 6 & 1 \\
\hline Chenopodiaceae & - & - & 32 & 27 \\
\hline Poaceae & - & - & - & 2 \\
\hline Caryophyllales & - & - & 273 & 709 \\
\hline Indeterminado & $2^{*}$ & $1^{*}$ & $1^{*}$ & 1 \\
\hline Total & 199 & 202 & 351 & 785 \\
\hline
\end{tabular}

Las especies recuperadas en los sitios coinciden con la distribución actual de plantas en el área de estudio, lo que estaría indicando que la vegetación no cambió sustancialmente en los últimos siglos. Si bien la actividad de animales fosoriales pudo haber homogeneizado la muestra eliminando diferencias temporales, no se determinaron especies que no puedan ser encontradas en la actualidad. Se observaron especies que aparecieron exclusivamente en el registro arqueológico, tal es el caso de Astragalus pehuenches en la excavación de GN-5 y Schinus polygamus y Berberis empetrifolia en la excavación de PJ-II. Las restantes especies estuvieron presentes tanto en los macrorrestos recuperados en la excavación como en los sondeos de prueba.

El taxón que refleja altos valores de densidad para todos los conjuntos analizados pertenece al orden Caryophyllales. La presencia de este orden tanto en los sitios arqueológicos como en los 
Tabla 5. Estado de conservación, estructural y densidad de los restos arqueológicos vegetales recuperados mediante flotación y zaranda de la excavación PJ-II.

Plant part, condition, structure and density of archaeological remains recovered by flotation from the archaeological site of PJ-II.

\begin{tabular}{|c|c|c|c|c|c|c|}
\hline Cuadrícula & Nivel & Taxón & Parte Vegetal & $\begin{array}{c}\text { Estado de } \\
\text { Conservación }\end{array}$ & $\begin{array}{c}\text { Estado } \\
\text { Estructural }\end{array}$ & $\begin{array}{c}\text { Densidad } \\
\left(\mathrm{N}^{\circ} / 5 \text { litros }\right)\end{array}$ \\
\hline & & Schinus polygamus & Endocarpo & Carbonizado & Entero & 0,8 \\
\hline & & Schinus polygamus & Endocarpo & Carbonizado & Frag. & \\
\hline & 3 & Chenopodium aff. papulosum & Semilla & Seco & Entero & 0,2 \\
\hline & & Caryophyllales & Semilla & Seco & Entero & 25,4 \\
\hline & & Schinus polygamus & Endocarpo & Carbonizado & Frag. & 0,4 \\
\hline & & Berberis empetrifolia & Semilla & Carbonizado & Entero & 0,2 \\
\hline & 4 & Chenopodium aff. papulosum & Semilla & Seco & entero & 2,2 \\
\hline & & Caryophyllales & Semilla & Seco & Entero & 19 \\
\hline & & Schinus polygamus & Endocarpo & Carbonizado & Entero & 2,2 \\
\hline & & Berberis empetrifolia & Endocarpo & Carbonizado & Frag. & 0,2 \\
\hline & 5 & Oxalis sp. & Semilla & Seco & Entero & 0,2 \\
\hline & & Caryophyllales & Semilla & Seco & Entero & 5,8 \\
\hline & & Chenopodiaceae & Semilla & Seco & Entero & 4,2 \\
\hline & & Schinus polygamus & Endocarpo & Carbonizado & Entero & 0,8 \\
\hline & 6 & Schinus polygamus & Endocarpo & Carbonizado & Frag. & \\
\hline & 0 & Caryophyllales & Semilla & Seco & Entero & 2,4 \\
\hline & & Chenopodiaceae & Semilla & Seco & Entero & 0,2 \\
\hline \multirow[t]{18}{*}{ A-1 } & & Schinus polygamus & Endocarpo & Carbonizado & Entero & 0,6 \\
\hline & & Schinus polygamus & Endocarpo & Carbonizado & Frag. & \\
\hline & & Caryophyllales & Semilla & Seco & Entero & 0,4 \\
\hline & & Chenopodiaceae & Semilla & Seco & Entero & 0,4 \\
\hline & 8 & Berberis empetrifolia & Semilla & Carbonizado & Entero & 0,2 \\
\hline & 0 & Caryophyllales & Semilla & Seco & Entero & 0,4 \\
\hline & & Schinus polygamus & Endocarpo & Carbonizado & Entero & 1,4 \\
\hline & 9 & Schinus polygamus & Endocarpo & Carbonizado & Frag. & \\
\hline & & Indeterminado & Semilla & Carbonizada & Entero & 0,2 \\
\hline & 10 & Schinus polygamus & Endocarpo & Carbonizado & Entero & 0,2 \\
\hline & & Caryophyllales & Semilla & Seco & Entero & 0,2 \\
\hline & & Schinus polygamus & Endocarpo & Carbonizado & Entero & 0,4 \\
\hline & 10 & Schinus polygamus & Endocarpo & Carbonizado & Frag. & \\
\hline & & Caryophyllales & Semilla & Seco & Entero & 0,2 \\
\hline & & Chenopodiaceae & Semilla & Seco & Entero & 0,2 \\
\hline & 15 & Schinus polygamus & Endocarpo & Carbonizado & Entero & 0,2 \\
\hline & 10 & Caryophyllales & Semilla & Seco & Entero & 0,2 \\
\hline & & Chenopodiaceae & Semilla & Seco & Entero & 0,8 \\
\hline
\end{tabular}


Continuación Tabla 5.

\begin{tabular}{|c|c|c|c|c|c|c|}
\hline Cuadrícula & Nivel & Taxón & Parte Vegetal & $\begin{array}{c}\text { Estado de } \\
\text { Conservación }\end{array}$ & $\begin{array}{c}\text { Estado } \\
\text { Estructural }\end{array}$ & $\begin{array}{c}\text { Densidad } \\
\left(\mathrm{N}^{\circ} / 5 \text { litros }\right)\end{array}$ \\
\hline \multirow{36}{*}{ A-2 } & \multirow{5}{*}{1} & Schinus polygamus & Endocarpo & Carbonizado & Entero & 0,4 \\
\hline & & Schinus polygamus & Endocarpo & Carbonizado & Frag. & \\
\hline & & Erodium cicutarium & Fruto & Seco & Entero & 0,6 \\
\hline & & Caryophyllales & Semilla & Seco & Entero & 32,4 \\
\hline & & Chenopodiaceae & Semilla & Seco & Entero & 0,8 \\
\hline & \multirow{4}{*}{3} & Schinus polygamus & Endocarpo & Carbonizado & Entero & 0,4 \\
\hline & & Schinus polygamus & Endocarpo & Carbonizado & Frag. & \\
\hline & & Caryophyllales & Semilla & Seco & Entero & 81,4 \\
\hline & & Chenopodiaceae & Semilla & Seco & Entero & 0,4 \\
\hline & \multirow{5}{*}{4} & Schinus polygamus & Endocarpo & Carbonizado & Entero & 1 \\
\hline & & Schinus polygamus & Endocarpo & Carbonizado & Frag. & \\
\hline & & Erodium cicutarium & Fruto & Seco & Entero & 0,2 \\
\hline & & Caryophyllales & Semilla & Seco & Entero & 17,6 \\
\hline & & Chenopodiaceae & Semilla & Seco & Entero & 1,8 \\
\hline & \multirow{4}{*}{5} & Schinus polygamus & Endocarpo & Carbonizado & Entero & 3 \\
\hline & & Schinus polygamus & Endocarpo & Carbonizado & Frag. & \\
\hline & & Caryophyllales & Semilla & Seco & Entero & 4,2 \\
\hline & & Chenopodiaceae & Semilla & Seco & Entero & 2,6 \\
\hline & \multirow{4}{*}{7} & Schinus polygamus & Endocarpo & Carbonizado & Entero & 1,2 \\
\hline & & Schinus polygamus & Endocarpo & Carbonizado & Frag. & \\
\hline & & Caryophyllales & Semilla & Seco & Entero & 3,8 \\
\hline & & Poaceae & - & - & Frag. & 0,2 \\
\hline & \multirow{2}{*}{10} & Schinus polygamus & Endocarpo & Carbonizado & Entero & 0,4 \\
\hline & & Caryophyllales & Semilla & Seco & Entero & 0,2 \\
\hline & \multirow{3}{*}{11} & Schinus polygamus & Endocarpo & Carbonizado & Entero & 0,8 \\
\hline & & Schinus polygamus & Endocarpo & Carbonizado & Frag. & \\
\hline & & Caryophyllales & Semilla & Seco & Entero & 0,4 \\
\hline & 12 & Caryophyllales & Semilla & Seco & Entero & 0,6 \\
\hline & \multirow{4}{*}{13} & Schinus polygamus & Endocarpo & Carbonizado & Entero & 0,8 \\
\hline & & Schinus polygamus & Endocarpo & Carbonizado & Frag. & \\
\hline & & Caryophyllales & Semilla & Seco & Entero & 0,4 \\
\hline & & Indeterminado & Semilla & Seco & Entero & 0,2 \\
\hline & \multirow{2}{*}{17} & Schinus polygamus & Endocarpo & Carbonizado & Entero & 0,2 \\
\hline & & Caryophyllales & Semilla & Seco & Entero & 0,2 \\
\hline & 18 & Caryophyllales & Semilla & Seco & Entero & 0,2 \\
\hline & 19 & Caryophyllales & Semilla & Seco & Entero & 0,4 \\
\hline
\end{tabular}



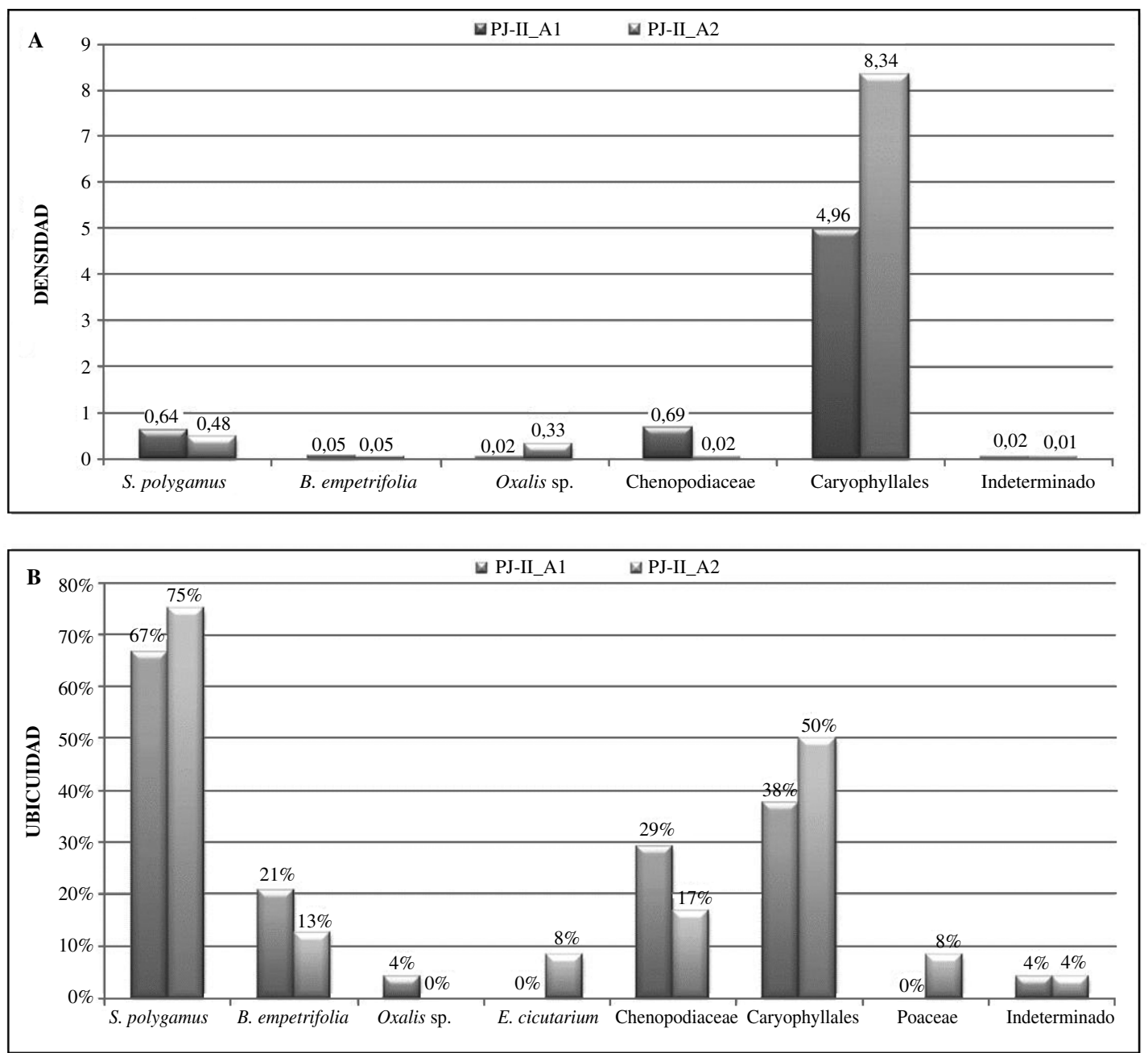

Figura 4. (A) Densidad de los macrorrestos vegetales en el sitio PJ-II; (B) ubicuidad de los macrorrestos vegetales en el sitio PJ-II. (A) Density of plant remains from PJ-II sites; (B) ubiquity of plant remains from PJ-II sites.

sondeos de prueba, sugiere que el origen de este taxón en el registro arqueológico se debe a causas naturales y no a un origen antrópico. Comparar las densidades de plantas puede ayudar a determinar el origen de los macrorrestos, además de permitir evaluar los movimientos de los restos de plantas en el sitio (Lennstrom y Hastorf 1995). En este sentido, excluyendo al taxón Caryophyllales, los valores más altos de densidad para el sitio PJ-II están reflejados por Schinus polygamus y Chenopodiaceae, y para el caso de GN-5 por Astragalus pehuenches. Lo anteriormente expuesto permite, junto con la evaluación de las otras variables, argumentar en favor de que la presencia de Schinus polygamus y Astragalus pehuenches tenga un origen antrópico.
La aparición de macrorrestos carbonizados (Schinus polygamus y Berberis empetrifolia) en el registro arqueológico de PJ-II pudo deberse a que estas especies formaron parte del consumo como alimento. Es probable que las mismas se hayan carbonizado accidentalmente durante alguna forma de preparación de los mismos. En comunidades actuales del sur argentino, los frutos de Berberis empetrifolia se comen crudos o son utilizados para hacer dulces o jaleas (Ladio 2002; Ladio y Lozada 2009). En Chile, la especie Schinus polygamus ha sido utilizada en la preparación de bebidas, como condimentos de comidas y para consumo en fresco (Ortiz Garmendia 1969). Además, la decocción de sus hojas ha sido utilizada para la tinción de lanas (Hernández 2002), esta especie ha sido reportada 

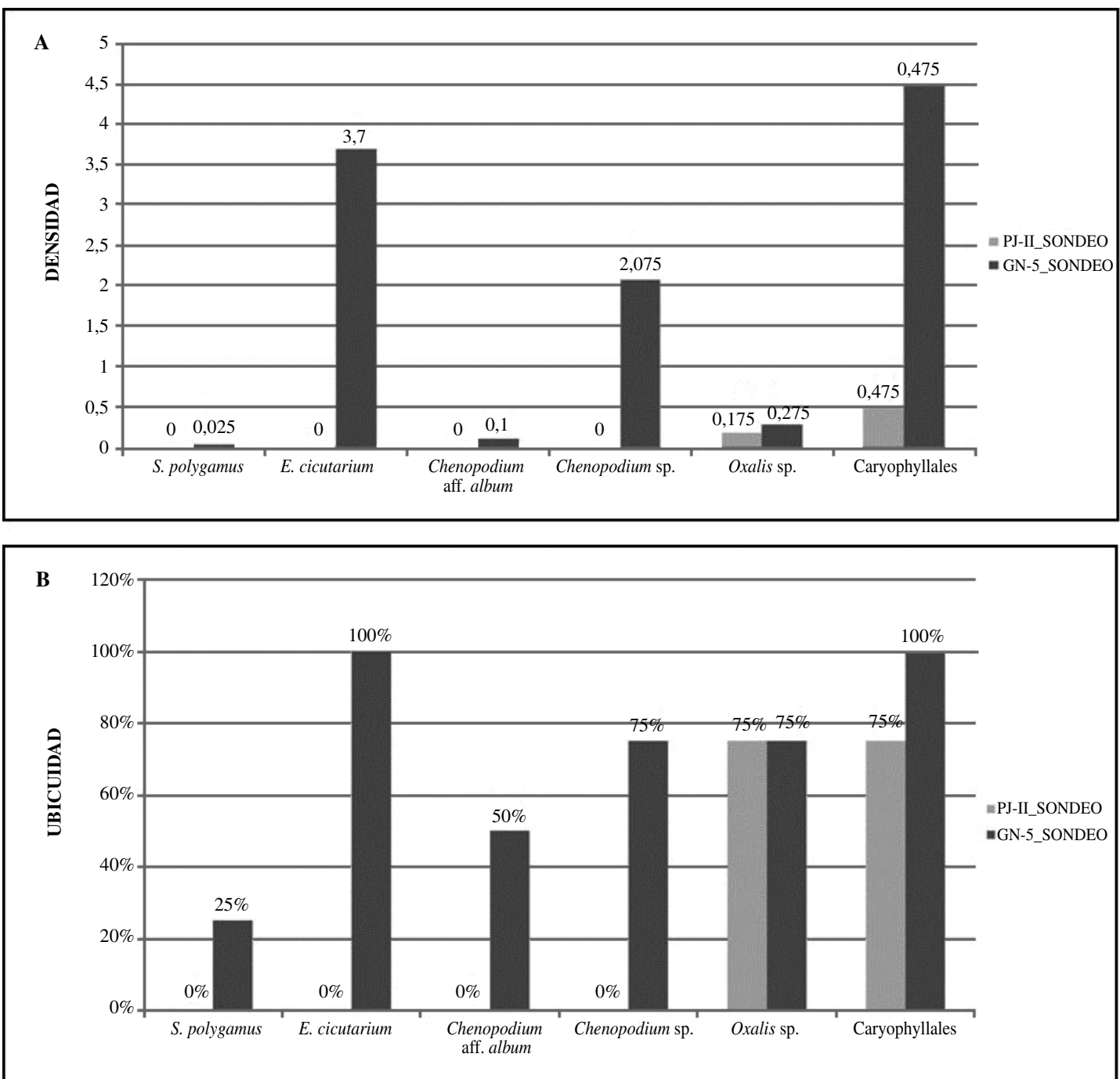

Figura 5. (A) Densidad de los macrorrestos vegetales recuperados de los sondeos de GN-5 y PJ-II; (B) ubicuidad de los macrorrestos vegetales recuperados de los sondeos de GN-5 y PJ-II.

(A) Density of plant remains recovered in the pit test from GN-5 and PJ-II sites; (B) ubiquity of plant remains recovered in the pit test from $G N-5$ and PJ-II sites.

en la medicina tradicional chilena para el lavado de heridas y dislocaciones de las articulaciones (Montes y Wilkomirsky 1986). Estudios sobre rendimiento económico de las plantas, que se están llevando a cabo en la actualidad, ubican a esta especie (Schinus polygamus) en segundo lugar en el ranking de recursos vegetales en el sur de Mendoza (Llano y Ugan 2010), lo que destaca su importancia potencial entre las poblaciones humanas.

Dado que estas especies cuentan con registros etnohistóricos de uso (Hernández 2002; Ladio 2001; Ladio y Lozada 2009; Pochettino 1988) y su estado de preservación es carbonizado, se puede inferir que en este lapso temporal la presencia de las especies mencionadas es de origen antrópico y de este modo revalorizar su presencia en el sitio arqueológico. En cuanto a los endocarpos carbonizados que se recuperaron de las galerías de animales fosoriales, se asume que los mismos también habrían ingresado al sitio arqueológico por el agente humano, dado que presentan el mismo estado, tanto estructural como de preservación, que los restos recuperados fuera de las mismas. Sin embargo, estos restos pueden presentar alteraciones en relación a su posición estratigráfica como producto de la remoción de los sedimentos 
por parte de estos animales. Un único endocarpo de Schinus polygamus fue hallado en el sondeo próximo a la excavación de PJ-II, éste se atribuye a depositación natural (i.e. lluvia natural de semillas, fecas de animales, entre otros). El mismo apareció en el nivel 1; su estado de preservación es seco, no manifiesta signos de uso antrópico y, además, esta especie se encuentra naturalmente ocupando parches densos en toda el área de estudio.

En GN-5 se da el caso contrario con Astragalus pehuenches, esta especie, a pesar de no presentar vestigios de uso (e.g. carbonizado), sus macrorrestos solo aparecieron en la excavación. De haber sido utilizada, la presencia de esta especie debería ser pensada en términos de otros usos no vinculados al consumo, ya que ha sido demostrado que su consumo, al menos en ganado ovino y equino, produce locoísmo (Robles et al. 2000), situación por la cual esta planta ha sido incluida en la lista de plantas tóxicas. No obstante, no se descarta su uso dado que es una de las pocas especies encontradas en la zona que puede ser utilizada como madera ya sea para la confección de artefactos o leña. En este sentido, los análisis antracológicos serán esclarecedores en cuanto a la presencia de esta especie en el sitio arqueológico. No ocurre lo mismo con los restantes taxa identificados, los cuales están presentes en ambas muestras, es decir, tanto en la excavación como en el sondeo de control. En general, los macrorrestos provenientes de la excavación de GN-5 y los recuperados en el sondeo permiten inferir que el conjunto total tenga más que ver con procesos de depositación natural que antrópicos, aunque la presencia de Astragalus pehuenches estaría indicando que al menos una parte de la muestra total podría no ser asignada a la depositación natural.

Las semillas de Oxalis sp. y Chenopodium spp. halladas en ambos sitios arqueológicos se encuentran en estado de preservación seco y en GN-5 fueron recuperadas tanto en la excavación del sitio como en el sondeo de control, por lo que es altamente probable un origen natural de depositación. No obstante, semillas de Chenopodium spp. no fueron recuperadas en el sondeo de PJ-II. En este sentido, es necesario tener en cuenta que los estudios etnobotánicos llevados adelante por diferentes investigadores sostienen que estas plantas han sido y son usadas en la actualidad (Gremillion 1996; Hunziker y Planchuelo 1971; Ladio 2002; Planella 1997; Planella y Tagle 1995; Rapoport et al. 1998; Tagle y Planella 2002), por lo que no es posible descartar el origen antrópico de esos macrorrestos y que por lo tanto futuros estudios en sitios adyacentes aporten otro tipo de información.

En resumen, las características del registro arqueobotánico de PJ-II (ubicuidad, estado de conservación, etc.) indicarían un origen cultural de la mayor parte de sus restos. Por el contrario, los mismos indicadores en $\mathrm{GN}-5$ sugieren la idea de que el principal aporte de macrorrestos al sitio arqueológico haya sido el contexto natural que rodea al mismo. Las diferencias en la presencia de elementos de molienda (alta frecuencia en PJ-II y nula en GN-5), así como los resultados de los sondeos de prueba estarían confirmando las tendencias mencionadas. Sin embargo, dada la falta de análisis de microrrestos (almidones y fitolitos), aún no es posible afirmar con seguridad que los elementos de molienda recuperados en el sitio PJ-II hayan sido utilizados para procesar los taxa recuperados durante las excavaciones.

Los resultados obtenidos muestran, por un lado, lo complejo de la conformación de este tipo de registro, mientras que por otro sugieren el uso de las plantas no solo como complemento de la caza, sino tal vez como una parte central en la dieta y subsistencia humana prehispánica de la región.

\section{Conclusión}

El presente trabajo ha abordado el análisis arqueobotánico de los sitios Gendarmería Nacional-5 y Puesto Jaque-II localizados en el valle del Salado. El mismo, permitirá discutir en mayor profundidad cuál pudo haber sido el rol de este tipo de recursos entre los grupos prehispánicos de la región durante los últimos mil años, momento para el cual se ha propuesto un uso mayor de los recursos vegetales (Neme 2007; Neme y Gil 2008).

Los temas desarrollados en este trabajo estuvieron vinculados a la formación del depósito, como así también a conocer cuáles fueron los taxa explotados y la importancia relativa de los mismos en cada sitio arqueológico. Los grupos humanos que habitaron el valle del Salado durante el Holoceno tardío habrían hecho uso de las especies vegetales locales, presentes actualmente en el área, seleccionando las mismas tanto para el consumo como para otros usos. La presencia de endocarpos carbonizados y especies con referencias etnobotánicas de uso sugieren que al menos una parte de ésta fue procesada antes de ser 
utilizada. La presencia de elementos de molienda (manos y molinos) en PJ-II reforzaría esta idea.

En cuanto al origen de los conjuntos, más allá del estado de preservación, seco o carbonizado, la presencia de especies tales como Astragalus pehuenches, Schinus polygamus, Berberis empetrifolia en la excavación y ausentes en los sondeos, permiten inferir que al menos parte de la muestra encontrada en los sitios arqueológicos sea de origen antrópico.

La alta frecuencia de macrorrestos, especialmente en PJ-II, sugiere que los recursos vegetales debieron tener un rol importante entre las poblaciones humanas que habitaron la cuenca del Salado. Esta primera conclusión contradice al menos en parte las ideas de que estos espacios de altura eran ocupados para hacer un uso casi exclusivo de los recursos animales que ascendían a la cordillera durante los momentos más cálidos del año (Gambier 1980; Lagiglia 1997). La alta cantidad de Schinus polygamus en el registro de PJ-II, la mayor parte de ellos termoalterados, confirma su importancia entre los grupos humanos del sur de Mendoza, dado que el mismo taxón ha sido recuperado en otros ambientes de la región (Durán 2004; Gambier 1980; Gil et al. 2006; Hernández et al. 1999). Las especies recuperadas en PJ-II indican que se utilizaron tanto plantas de alto retorno energético (Schinus) como de retorno bajo (Chenopodium), lo que sugiere que más allá de los escasos taxa recuperados la amplitud de dieta pudo ser importante en relación a los recursos vegetales de la región.

A diferencia de lo que ocurriría en PJ-II, el caso de GN-5 puede constituirse en un buen ejemplo de la convergencia de diferentes agentes de depositación en el registro arqueológico, en este caso tal vez debido a la contribución natural de las plantas durante su proceso de dispersión. Como se mencionó anteriormente, dada la menor cantidad de macrorrestos, la no carbonización de los mismos, los tipos de taxa recuperados y su similitud con los sondeos de prueba en términos de densidad y ubicuidad, su vinculación con el uso humano es poco probable.

Por último, si bien no concluyente, el registro arqueobotánico aquí estudiado concuerda con el mayor uso esperado de las plantas para los últimos 2000 años tal como había sido propuesto en el modelo de intensificación propuesto para la región (Neme 2007; Neme y Gil 2008). Consideramos que si bien este trabajo incluye solo dos sitios dentro de una amplia región de estudios, el mismo constituye un primer aporte a la discusión de los procesos de formación del registro arqueológico en general y arqueobotánico en particular, así como al uso humano de las plantas en las áreas de cordillera del sur de Mendoza.

Agradecimientos: Agradecemos a María Lelia Pochettino y Adolfo Gil quienes colaboraron con sugerencias y comentarios para mejorar el manuscrito. A María Fernanda Rodríguez, Iris Peralta y Roberto Kiesling quienes colaboraron con la determinación taxonómica. A Sergio Etcheverri y Luis Ballarini por su desinteresada colaboración. Este trabajo se realizó en el marco de proyectos subsidiados por la Agencia Nacional de Promoción Científica y Tecnológica, PICT -2007-00610 y PICT 04-12750 (2005-2006). A la empresa Valle de Las Leñas S.A. por su colaboración en los trabajos de campo. Por último, a los evaluadores que permitieron mejorar sustancialmente la calidad del presente manuscrito.

\section{Referencias Citadas}

Alonso Martínez, N., J. Juan Tresserras, M.O. Rodríguez-Arisa y N. Rovira Buendía 2003. Muestreo Arqueobotánico de Yacimientos al aire libre y en medio seco. En La Recogida de Muestras en Arqueobotánica: Objetivos y Propuestas Metodológicas. La Gestión de los Recursos Vegetales y la Transformación del Paleopaisaje en el Mediterráneo Occidental, dirigido por R. Buxó y R. Piqué, pp. 31-48. Editorial CEGE S.A., Barcelona.

Badal, E., Y. Carrión, D. Rivera y P. Uzquiano 2003. La arqueobotánica en cuevas y abrigos: objetivos y métodos de muestreo. En La Recogida de Muestras en Arqueobotánica: Objetivos y Propuestas Metodológicas. La Gestión de los Recursos Vegetales y la Transformación del Paleopaisaje en el Mediterráneo Occidental, dirigido por R. Buxó y R. Piqué, pp. 19-29. Editorial CEGE S.A., Barcelona.

Böcher, T.W., J.P. Hjerting y K. Rahn 1972. Botanical studies in the Atuel Valley area, Mendoza Province, Argentina. Part III. Dansk Botanisk Arkiv 22:195-358.

Buxó, R. 1997. Arqueología de las Plantas. Editorial Crítica, Barcelona.

Cabrera, A. 1976. Regiones fitogeográficas argentinas. En Enciclopedia Argentina de Agricultura y Jardinería, editado por W.F. Kugler Tomo 2, Fasc. 1, pp. 1-85. 2a edición. Acme, Buenos Aires. 
Capitanelli, R. 2005. Climas locales de la extremidad sur de las montañas. En Climatología de Mendoza, editado por R. Capitanelli, pp. 45-57. Facultad de Filosofía y Letras, Universidad Nacional de Cuyo, Mendoza.

Cowan, C.W. 1997. Evolutionary changes associated with the domestication of Cucurbita pepo: Evidence from Eastern Kentucky. En People, Plants and Landscapes. Studies in Paleoethnobotany, editado por K.J. Gremillion, pp. 63-85. Alabama Press, Tuscaloosa.

Durán, V. 2004. Poblaciones Indígenas de Malargüe. Su Arqueología e Historia. Ceider, Serie de Libros $\mathrm{N}^{\circ} 1$. Facultad de Filosofía y Letras, Universidad Nacional de Cuyo, Mendoza.

Gambier, M. 1980. Excavaciones en la Gruta de El Manzano, Río Grande-Mendoza. Boletín del Museo de Ciencias Naturales y Antropológicas Juan Cornelio Moyano 1:45-55. Mendoza.

Gil, A. y G. Neme (eds.) 2002. Entre Montañas y Desiertos: Arqueología del Sur de Mendoza. Sociedad Argentina de Antropología, Buenos Aires.

Gil, A., R. Tykot, G. Neme y N. Shelnut 2006. Maize on frontier isotopic and macrobotanical data from Central-Western Argentina. En Histories of Maize. Multidisciplinary Approaches to the Prehistory, Biogeography, Domestication, and Evolution of Maize, editado por J. Staller, R. Tykot y B. Benz, pp. 199-214. Academic Press, San Diego.

Giovannetti, M., A. Capparelli y M.L. Pochettino 2008. La arqueobotánica en Sudamérica. ¿Hacia un equilibrio de enfoques? Discusión en torno a las categorías clasificatorias y la práctica arqueobotánica y paleoetnobotánica. En Arqueobotánica y Teoría Arqueológica. Discusiones desde Suramérica, editado por S. Archila, M. Giovannetti y V. Lema, pp. 17-34. Uniandes -CESO, Bogotá

Gremillion, K.J. 1993. The evolution of seed morphology in domesticated Chenopodium: An archaeological case study. Journal of Ethnobiology 13:149-169.

_ _ _ 1 1996. Diffussion and adoption of crops in evolutionary perspective. Journal of Anthropological Archaeology 15:183-204.

- _ - 1997. Introduction. En People, Plants and Landscapes. Studies in Paleoethnobotany, editado por K.J. Gremillion, pp. 1-3. Alabama Press, Tuscaloosa.

Hastorf, C. 1999. Recent research in paleoethnobotany. Journal of Archaeological Research 7:55-103.

Hastorf, C. y V. Popper (eds.) 1988. Current Paleoethnobotany: Analytical Methods and Cultural Interpretations of Archaeological Plant Remains. The University of Chicago Press, Chicago.

Hernández, A. 2002. Paleoetnobotánica en el Sur de Mendoza. En Entre Montañas y Desiertos Arqueología del Sur de Mendoza, editado por A. Gil y G. Neme, pp. 157-180. Sociedad Argentina de Antropología, Buenos Aires.

Hernández, A., H. Lagiglia y A. Gil 1999. El registro arqueobotánico en el Sitio Agua de los Caballos-1 (San Rafael, Mendoza). Anales de Arqueología y Etnología 54-55:181-203.

Hunziker, A y A. Planchuelo 1971. Sobre un nuevo hallazgo de Amaranthus caudatus en tumbas indígenas de Argentina. Notas del Museo N13, Kurtiziana, Vi. 63-67. Córdoba.
Johannessen, S. 1988. Plant remains and culture change: Are paleoethnobotanical data better than we think? En Current Paleoethnobotany: Analytical Methods and Cultural Interpretations of Archaeological Plant Remains, editado por C. Hastorf y V. Popper, pp. 145-166. The University of Chicago Press, Chicago.

Jones, M. 1991. Sampling in paleoethnobotany. En Progress in Old World Paleoethnobotany, editado por W. van Zeist, K. Wasylikowa y K.-E. Behre, pp. 63-80. Balkema, Rotterdam.

Keepax, C. 1977. Contamination of archaeological deposits by seeds of modern origin with particular reference to the use of flotation machines. Journal Archaeological Science 4:221-229.

Ladio, A. 2001. The maintenance of wild edible plant gathering in a mapuche community of Patagonia. Economic Botany $55: 243-254$

_ _ _ 2002. Las plantas comestibles en el noroeste patagónico y su utilización por las poblaciones humanas: una aproximación cuantitativa. Tesis Doctoral, Universidad Nacional del Comahue, Neuquén.

Ladio, A. y M. Lozada 2009. Human ecology, ethnobotany and traditional practices in a rural population of the Monte region, Argentina: Resilience and ecological knowledge. Journal of Arid Environments 73:222-227.

Lagiglia, H. 1997. Arqueología prehistórica del Atuel y del Diamante. Revista del CINTER 2:29-46.

Lagiglia, H., A. Gil y G. Neme 1994. Investigaciones arqueológicas en la Cueva Arroyo Colorado. Actas y Memorias del XI Congreso Nacional de Arqueología Argentina Tomo 2:119-120. San Rafael, Mendoza.

Lennstrom, H. y C. Hastorf 1995. Interpretation in context: Sampling and analysis in paleoethnobotany. American Antiquity 60:701-721.

Llano, C. 2008. El registro arqueobotánico en el sitio Arroyo Malo-3, alto valle del Atuel, Mendoza, Argentina. Intersecciones en Antropología 9:133-143.

Llano, C. y A. Ugan 2010. Rendimiento económico de plantas silvestres del sur de Mendoza: valores nutricionales, costos de manejo e interpretación del registro arqueológico. En Tradiciones y Transformaciones en Etnobotánica, editado por M.L. Pochettino, A.H. Ladio y P.M. Arenas, pp. 44-48. RisapretCyted, La Plata-Buenos Aires.

Martin, A. y W. Barkley 1973. Seed Identification Manual. University of California Press, California.

Miksicek, C. 1987. Formation of the archaeobotanical record. Advances in Archaeological Method and Theory 10:211-247.

Miller, N. 1988. Ratios in paleoethnobotanical analysis. En Current Paleoethnobotany: Analytical Methods and Cultural Interpretations of Archaeological Plant Remains, editado por C. Hastorf y V. Popper, pp. 72-85. The University of Chicago Press, Chicago.

Minnis, P. 1978. Paleoethnobotanical indicators of prehistoric environmental disturbance: A case of study. En The Nature and Status of Ethnobotany, editado por. R. Ford, M.F. Brown, M. Hodge y W.L. Merrill, pp. 347-366. Anthropological papers 67. University of Michigan, Museum of Anthropology. 
_. _ 1981. Seeds in archaeological sites: sources and some interpretative problems. American Antiquity 46:143-152.

Montes, M. y T. Wilkomirsky 1986. Medicina Tradicional Chilena. Editorial de la Universidad de Concepción, Santiago.

Neme, G. 2007. Cazadores-recolectores de altura en los Andes Meridionales. British Archaeological Reports International Series 1591. Archaeopress, Oxford.

Neme, G y A. Gil 2008. Faunal exploitation and agricultural transitions in the South American Agricultural limit. International Journal Osteoarchaeology. Published online in Wiley InterScience (www.interscience.wiley.com)

_ _ _ 2008. Biogeografía humana en los Andes Meridionales: tendencias arqueológicas en el sur de Mendoza. Chungara Revista de Antropología Chilena 40:5-18.

Novellino, P. y G. Neme 1999. Hallazgos de partes esqueletales humanas en Laguna Blanca y El Sosneado (Sur de Mendoza). Actas del XII Congreso Nacional de Arqueología Argentina. Tomo II, pp. 256-260. La Plata.

Ortiz Garmendia, J. 1969. Plantas Silvestres con Frutos Comestibles por el Hombre (Contribuciones Arqueológicas 8). Museo Arqueológico, La Serena.

Pearsall, D. 1989. Paleoethnobotany. A Handbook of Procedures. Academic Press, inc. San Diego.

Pérez Winter, C. 2008. Organización Tecnológica durante el Holoceno Medio en el Alto Valle del Río Atuel, Sur de Mendoza: Caso Arroyo Malo 3. Tesina de Licenciatura, Universidad de Buenos Aires, Buenos Aires.

Planella, T. 1997. Restos alimenticios de origen vegetal en el contexto habitacional litoral de la Cultura Aconcagua. Manuscrito en posesión del autor.

Planella, T. y B. Tagle 1995. La Quinoa (Chenopodium quinoa Willd.) un aporte alimenticio ancestral hoy desconocido. Gastroenterología Latinoamericana 6:70-83.

Pochettino, M.L. 1988. Diferenciación de los frutos de los "molles" de importancia etnobotánica en el Noroeste Argentino. Notas del Museo de La Plata. Botánica 21 (101):159-167.
Pochettino, M.L. y M.C. Scattolin 1991. Identificación y significado de frutos y semillas carbonizados de sitios arqueológicos formativos de la ladera occidental del Aconquija (Catamarca, República Argentina). Revista del Museo de La Plata: Sección Antropología 9 (71):169-181.

Popper, V. 1988. Selecting quantitative measurements in paleoethnobotany. En Current Paleoethnobotany: Analytical Methods and Cultural Interpretations of Archaeological Plant Remains, editado por C. Hastorf y V. Popper, pp. 53-71. The University of Chicago Press, Chicago.

Rapoport, E., A. Ladio, E. Raffaele, L. Ghermandi y E. Sanz 1998. Malezas comestibles. Hay yuyos y yuyos.... Ciencia Hoy 9:30-43. Buenos Aires.

Robles, C.A., C. Saber y M. Jefrey 2000. Intoxicación por Astragalus pehuenches (locoísmo) en ovinos Merino de la Patagonia Argentina. Revista de Medicina Veterinaria 81:380-384.

Rodríguez, F. 1999. Plant species (Poaceae, Asteraceae, Fabaceae and Solanaceae) at an archaeological site in the southern Argentine Puna. Journal of Ethnobiology 19:228-247.

. _. - 2005. Human evidence from the mid-Holocene in the salty Argentine Puna: Analysis of the archaeological record. Quaternary International 132:15-22.

Roig, F. 1972. Bosquejo Fisonómico de la vegetación de la Provincia de Mendoza. Boletín de la Sociedad Argentina de Botánica 13 (Suplemento): 49-80. La Plata.

Roig, F., E. Martínez Carretero y E. Méndez 2000. Vegetación de la provincia de Mendoza. En Argentina Recursos y Problemas Ambientales de la Zona Árida, editado por E. Abraham y F. Rodríguez Martínez, pp. 63-64. Programa de cooperación para la Investigación, Junta de Gobierno de Andalucía-Universidades y Centros de Investigación de la Región Andina Argentina, Mendoza.

Schobinger, J. 1975. La Prehistoria y Protohistoria de la Región Cuyana. Museo de Ciencias Naturales y Antropológicas Juan Cornelio Moyano, Mendoza.

Tagle, B. y T. Planella 2002. La Quinoa en la Zona Central de Chile. Supervivencia de una Tradición Prehispana. IKU, Santiago. 
